\title{
Factores asociados a la no adherencia al tratamiento antirretroviral de gran actividad en adultos infectados con el VIH-sida
}

\author{
Factors associated with non-adherence to highly active antiretroviral treatment in \\ HIVIAIDS patients
}

\author{
Óscar Alvis ${ }^{1}$, Liz De Coll ${ }^{1}$, Lourdes Chumbimune ${ }^{1}$, Cristina Díaz $^{1}$, Judith Díaz ${ }^{1}$, Mary Reyes $^{2}$ \\ ${ }^{1}$ Estudiante de Medicina Humana, Facultad de Medicina, Universidad Nacional Mayor de San Marcos, Lima, Perú. \\ ${ }^{2}$ Médico epidemiólogo, Departamento Académico de Medicina Preventiva y Salud Pública, Facultad de Medicina, Universidad Nacional Mayor de San Marcos, Lima, Perú.
}

\begin{abstract}
Resumen
Introducción: La no adherencia al tratamiento antirretroviral de gran actividad (Targa) es la principal causa de fracaso terapéutico. Objetivos: Determinar la prevalencia y los factores asociados a la no adherencia al Targa en adultos infectados con el VIH-sida. Diseño: Estudio transversal. Institución: Servicio de Infectología, Hospital Nacional Arzobispo Loayza. Participantes: Pacientes infectados con el VIH que se encontraban recibiendo tratamiento antirretroviral. Intervenciones: A pacientes infectados con el VIH que se encontraban recibiendo tratamiento antirretroviral en el hospital, se aplicó un cuestionario estructurado, elaborado en función a instrumentos que evalúan la adherencia y factores asociados. Para determinar la asociación, se empleó las pruebas de chi cuadrado y t de student, se estableció un nivel de significación estadística $p<0,05$, y para calcular el riesgo se utilizó el OR, con intervalos de confianza de $95 \%$. Se realizó análisis de regresión logística. Principales medidas de resultados: No adherencia al tratamiento antirretroviral de gran actividad. Resultados: Se encuestó 465 personas; la edad promedio fue $36,8 \pm 9,1$ años; $64,1 \%$ era varón. El $35,9 \%$ de los encuestados resultó ser no adherente. Los factores independientemente asociados a la no adherencia fueron: ser homosexual/bisexual (OR: 3,85, IC95\% 1,98 a 7,51), tener una baja calidad de vida relacionada a la salud (OR: 6,22, IC95\% 3,47 a 11,13), poco apoyo social (OR: 5,41 , IC95\% 3,17 a 9,22), no tener domicilio fijo (OR 3,34, IC95\% 1,93 a 5,79), tener morbilidad psíquica (OR 2,93, IC95\% 1,78 a 4,82) y tener mayor tiempo en tratamiento (OR 1,04, IC95\% 1,02 a 1,07). Conclusiones: La prevalencia de no adherencia fue mayor a la reportada previamente en este mismo hospital, pero similar a la encontrada en diferentes estudios, pese a la heterogeneidad de los mismos. Los factores de tipo psicosocial fueron los que influyeron de forma más importante en la falta de adherencia al tratamiento antirretroviral.

Palabras clave: Adherencias; agentes antirretrovirales; infecciones por VIH; síndrome de inmunodeficiencia adquirida.
\end{abstract}

\begin{abstract}
Introduction: Non-adherence to highly active antiretroviral treatment (HAART) is the principal reason of therapeutic failure. Objectives: To determine the prevalence and factors associated with non-adherence to HAART in HIV/AIDS patients. Design: Cross-sectional study. Setting: Infectious diseases service, Arzobispo Loayza National Hospital, a teaching hospital. Participants: HIV-infected patients on treatment with highly active antiretroviral treatment. Interventions: HIV-infected patients on treatment with highly active antiretroviral treatment were invited to complete a constructed questionnaire based on instruments evaluating adherence and associated factors. $x^{2}$-test and Student's $t$ test were used to determine association; $p$-values below 0,05 were considered significant. Association magnitude was measured by $\mathrm{OR}$ and $95 \%$ confidence intervals. A multivariate logistic regression analysis was performed. Main outcome measures: Non-adherence to highly active antiretroviral treatment. Results: Four hundred and sixty five patients were interviewed, median age 36,8 $\pm 9,1$ years, $64,1 \%$ males. Nearly $35,9 \%$ of the sample was considered non-adherent. In multivariate analysis, being homosexual/bisexual (OR 3,85, 95\% Cl 1,98-7,51), having a low health-related quality of life $(\mathrm{OR} 6,22,95 \% \mathrm{Cl} 3,47-11,13)$, low social support (OR 5,41, 95\% Cl 3,17-9,22), not having a permanent residence (OR 3,34, $95 \% \mathrm{Cl} 1,93-5,79)$, having psychological morbidity (OR 2,93, 95\% Cl 1,78-4,82) and having received treatment for a long time $(\mathrm{OR} 1,04,95 \% \mathrm{Cl} 1,02-1,07)$ were independently associated with non-adherence. Conclusions: The prevalence of non-adherence was higher than the previously reported in this hospital, but similar to many studies despite their heterogeneity. Mainly psychosocial factors influenced non-adherence to antiretroviral treatment.
\end{abstract}

Key words: Adherences; anti-retroviral agents; HIV infections; acquired immunodeficiency syndrome.

\section{INTRODUCCIÓN}

La epidemia del VIH/sida se ha convertido en uno de los más importantes problemas de salud pública del presente siglo. Los avances logrados en los últimos años han permitido conocer mejor la magnitud del problema, tanto en el diagnóstico de laboratorio, el tratamiento antirretroviral y mayor acceso a los programas eficaces de tratamiento y prevención ${ }^{(1,2)}$. El Programa Conjunto de las Naciones Unidas sobre el VIH/sida (ONUSIDA) y la Organización Mundial de la Salud (OMS), señalan que durante el año
2007 las nuevas infecciones por VIH adquiridas fueron 2,5 millones y que 2,1 millones de personas fallecieron por esta enfermedad, estimándose que a finales del 2007 el número de personas que vivían con el VIH alcanzó aproximadamente a 33,2 millones en todo el mundo ${ }^{(2)}$. En el Perú, la Dirección General de Epidemiología (DGE) del Ministerio de Salud ha registrado la notificación de 20610 casos de SIDA y 30389 infecciones por VIH hasta diciembre del año $2007^{(3)}$. La Dirección de Salud Lima Ciudad reportó en el año 2004 que el distrito que registró la mayor incidencia distrital anual de los casos VIH/sida (49,55/100 mil hab.) fue Lima Cercado ${ }^{(4)}$.

En el campo del tratamiento de la infección por VIH/sida han surgido avances notables, lo que ha representado un aumento de la supervivencia, mejoría de la calidad de vida de las personas con dicha infección y disminución de las complicaciones asociadas ${ }^{(5,6)}$. Con la introducción del tratamiento antirretroviral de gran actividad (Targa) se ha reducido significativamente las tasas de mortalidad y morbilidad asociadas a la infección por VIH y al sida, convirtien- 
do a esta infección en una enfermedad crónica tratable y ya no una enfermedad terminal ${ }^{(7,8)}$. A pesar que el Targa mejora la respuesta clínica, inmunológica y viral del paciente, es sumamente necesario mantener un gran nivel de adherencia a la terapia prescrita ${ }^{(9)}$, ya que de no mantenerla se produciría un incremento de la actividad viral y con ello se podría desarrollar rápidamente resistencia a los fármacos ${ }^{(10,11)}$. Por lo tanto, la adherencia cumple un papel clave en garantizar la efectividad de los fármacos, habiéndose demostrado que una incorrecta adherencia se relaciona con el aumento de los ingresos hospitalarios, la evolución a sida, y es la principal causa de fracaso terapéutico ${ }^{(12,13)}$.

La adherencia al Targa se define como el grado de interacción entre los servicios de salud y el usuario que contribuya al cumplimento del tratamiento y las indicaciones terapéuticas para el Targa, en una comunidad determinada, e implica un papel activo por parte del paciente, con una actitud positiva y un compromiso del mismo en la decisión de iniciar y mantener el tratamiento antirretroviral (6). Hoy en día, debido a los problemas sanitarios y económicos, la falta de adherencia a los antirretrovirales es considerado un problema de salud pública, ya que no permite llegar a la efectividad clínica, teniendo los recursos para el tratamiento (14). Desde la aparición del Targa, se ha llevado a cabo múltiples estudios con el fin de determinar avances en cuanto a la adherencia y determinar los factores relacionados con esta, así como evaluar las consecuencias clínicas derivadas de la falta de cumplimiento ${ }^{(15,16)}$.

Existen diversos métodos para la valoración de la adherencia. El método directo (concentraciones plasmáticas de fármacos antirretrovirales) es considerado el más objetivo; sin embargo, presenta limitaciones, debido al comportamiento cinético de los fármacos. Además, el establecimiento de un umbral estándar para determinar adherencia resulta cuestionable y requiere técnicas analíticas caras y complejas, por lo que no es aplicable de manera rutinaria en la mayoría de los hospitales ${ }^{(12)}$. Entre los métodos indirectos tenemos: la valoración del profesional sanitario, sistemas de control electróni- co de apertura de los envases (MEMS), recuento de medicación, registros de dispensación y cuestionarios. Los MEMS son dispositivos de control electrónico que contienen un microprocesador que registra hora y día en que se ha abierto el envase; es el método más fiable, teniendo una alta correlación con la efectividad del tratamiento ${ }^{(9)}$ y ha servido como referencia para establecer la validez de otros métodos ${ }^{(17)}$; sin embargo, su uso implica un costo económico alto ${ }^{(18)}$, por lo que su empleo queda restringido al ámbito de la investigación clínica y la validación de otros métodos ${ }^{(12)}$. El recuento de medicación ha sido utilizado con éxito, debido a que es poco costoso, permite una medida cuantitativa, es objetivo y relativamente poco sofisticado, aunque se ha observado que produce sobreestimación de la adherencia, cuando comparado con el sistema MEMS ${ }^{(19)}$. A pesar de ello, por las características descritas ha sido usado frecuentemente como patrón ${ }^{(15)}$. En el registro de dispensación, se ha observado una buena correlación con los resultados virológicos y aceptable especificidad y sensibilidad, resultando un método recomendable en su aplicación rutinaria; su principal limitación es que la dispensación de la medicación no es sinónimo de cumplimiento correcto. El uso de cuestionarios es un sistema sencillo, económico y asequible, demostrándose una correlación entre la adherencia autorreferida y la efectividad antirretroviral; también, se ha puesto de manifiesto su sensibilidad relativamente baja y una especificidad aceptable, al compararlo con métodos más objetivos; son métodos especialmente útiles si el individuo es identificado como no adherente (19). Entre los cuestionarios validados, cabe destacar el cuestionario SMAQ (simplified medication adherence questionnaire) (17). Diferentes estudios han contribuido en la validación, análisis de las limitaciones y determinación de la especificidad y sensibilidad de los distintos métodos; a pesar de ello, la recomendación actual es la de utilizar varios métodos asociados, para lograr una estimación de la adherencia con la mayor exactitud posible ${ }^{(12)}$.

La mayoría de los estudios ofrece resultados en los que solo 50 a $80 \%$ de los pacientes presentan buena adherencia al tratamiento, y se obtiene una buena correlación entre adherencia y control de la infección por el VIH, medida por la carga viral. Se observa que con adherencias superiores a $90 \%$ se obtiene un buen control virológico en 45 a 100\% de los pacientes. Por lo tanto, se señala que con los tratamientos antirretrovirales evaluados son necesarias adherencias estrictas (superiores a 90\%) para conseguir los objetivos terapéuticos ${ }^{(15,20)}$, llegando a obtener menos de 400 copias del virus con este último valor ${ }^{(7)}$. Es así que el criterio médico acordado para la adherencia al tratamiento es cuando este se cumple en 90 a $95 \%{ }^{(16,21)}$, verificado con el recuento viral y de linfocitos CD4.

En los últimos años, se ha publicado trabajos que estudian diferentes factores asociados a la adherencia. Sin embargo, se ha hecho difícil la generalización de estos resultados, debido a que difieren en el método utilizado para medir la adherencia, los factores evaluados, la población estudiada y el diseño del estudio. En forma general, los factores estudiados pueden ser clasificados en cuatro grandes grupos: a) los relacionados con el individuo (edad, sexo, nivel escolar, entorno familiar y social, etc.); b) los debidos a la enfermedad en sí misma; c) los propios del tratamiento antirretroviral (número de fármacos empleados, posología y efectos adversos); y, d) los relacionados con el equipo asistencial ${ }^{(5,6,12)}$. Los factores que han demostrado estar relacionados con la adherencia de una forma más relevante incluyen la complejidad del tratamiento, los efectos secundarios, los problemas psicológicos, la adicción activa a drogas y/o alcohol, la falta de soporte social y familiar y las actitudes y creencias del paciente acerca del tratamiento. Cabe destacar que, excepto por algunos factores que han sido asociados con una adherencia incorrecta en varios estudios, los resultados son frecuentemente no concluyentes o contradictorios ${ }^{(12,15)}$. Por ello, resulta relevante el estudio de los factores asociados a la adherencia del Targa, especialmente en nuestro país, donde la información al respecto es mínima, y de esta manera poder crear estrategias e intervenciones, con el fin de mejorar la adherencia y por consiguiente la calidad de vida del paciente que padece de la infección VIH/sida. 
Al mes de noviembre del 2005, en el Perú existían 7000 personas viviendo con el VIH-sida que necesitaban tratamiento, de las cuales aproximadamente 6298 $(89,97 \%)$ ya recibían Targa para tal fecha ${ }^{(22)}$. En nuestro medio, solo se encontró un estudio realizado en el Hospital Nacional Arzobispo Loayza, donde se halló 80,2\% de pacientes adherentes al tratamiento antirretroviral de gran actividad, pero no se buscó asociación con alguno de los factores mencionados ${ }^{(23)}$.

El objetivo principal del presente estudio fue determinar la prevalencia y los factores asociados a la no adherencia al Targa en los pacientes adultos con VIH que recibían dicho tratamiento en el Hospital Nacional Arzobispo Loayza (HNAL).

\section{MÉTODOS}

Se realizó un estudio analítico observacional transversal. La población diana estuvo constituida por todos los pacientes infectados por el VIH que se encontraban recibiendo Targa en el servicio de Infectología del Hospital Nacional Arzobispo Loayza (HNAL), registrados hasta el año 2007. Se tuvo como criterios de inclusión ser paciente infectado por el VIH mayor de 18 años y tener como mínimo un mes en Targa. Como criterios de exclusión, se consideró a pacientes gestantes infectadas con el virus del VIH y pacientes en estado grave y/o enfermedad terminal. Para una prevalencia esperada de $19,2 \%$, se calculó el tamaño de muestra de 449 , con el programa EPIDAT 3,1. Se efectuó un muestreo por conveniencia, invitándose a participar a los pacientes infectados con el $\mathrm{VIH}$ en Targa que asistieron al servicio de Infectología del HNAL durante los meses de julio a diciembre de 2007. Se tuvo como variable dependiente la adherencia al Targa y las variables independientes fueron sexo, edad, estado civil, grado de instrucción, ocupación, domicilio fijo, apoyo social, calidad de vida relacionado a la salud (CVRS), uso activo de drogas, orientación sexual, morbilidad psíquica, número total de pastillas, frecuencia de dosis, tiempo de tratamiento, síntomas o molestias relacionadas con el tratamiento, el motivo de no adherencia referido por los pacientes.
Para evaluar la adherencia se utilizó el cuestionario de adherencia SMAQ (simplified medication adherence questionnaire) y la evaluación contenida en el Registro de dispensación del servicio de Infectología del HNAL. Se consideró como no adherentes al Targa a los pacientes clasificados como tal por al menos uno de los métodos aplicados. El cuestionario SMAQ contiene 6 preguntas con respuesta cerrada; en función de las respuestas se valora el grado de adherencia; es un cuestionario validado en la población española y aplicado en nuestro medio; presenta $72 \%$ de sensibilidad y $91 \%$ de especificidad ${ }^{(17)}$. El cuestionario es dicotómico; cualquier respuesta en el sentido de no adherente fue considerado como que el encuestado era no adherente. Para evaluar el apoyo social, utilizamos el Cuestionario de apoyo social funcional de Duke-UNC-11, el cual consta de 11 ítems que evalúan el apoyo social funcional o cualitativo percibido (posibilidad de contar con personas para comunicarse y recibir demostraciones de amor, cariño y empatía). Cada ítem es puntuado en un gradiente de frecuencia que va desde 1 "Mucho menos de lo que deseo" a 5 "Tanto como deseo". La puntuación total se obtiene sumando las respuestas de cada ítem, estableciéndose el punto de corte de 'bajo apoyo social' en una puntuación igual o inferior a 32. Para evaluar la morbilidad psíquica se utilizó el Cuestionario de salud general de Goldberg (GHQ-12), instrumento diseñado para evaluar síntomas emocionales, bienestar psicológico o trastornos mentales comunes en la población general (ansiedad y depresión, disfunción social y pérdida de la confianza y autoestima). Consta de 12 ítems de tipo Likert, con 4 categorías. A las 4 categorías de respuesta se les adjudicó el valor 0 (respuestas 1 y 2) o 1 (respuestas 3 y 4); una puntuación mayor e igual a 3 determina la presencia de morbilidad psíquica. Para la CVRS, se usó el Índice de calidad de vida de Mezzich y Cohen, el cual es un instrumento autoaplicado validado para el Perú que incluye 10 ítems, que evalúan el bienestar físico, psicológico, emocional y la calidad de vida global. El indicador de la variable calidad de vida es cuantitativo, con valores que van de 10 hasta 100 , definiéndose en nivel bajo de calidad de vida un puntaje de 10 a 60 y nivel alto de calidad de vida un puntaje de 60 a 100 . Se elaboró preguntas para obtener los datos sobre sexo, edad, estado civil, grado de instrucción, ocupación, domicilio fijo, uso de drogas, orientación sexual, síntomas o molestias relacionadas con el tratamiento, motivo de no adherencia.

En base a los instrumentos señalados y a las preguntas elaboradas, se diseñó un cuestionario estructurado, que constaba de las siguientes partes:

- Preguntas 1 al 9, 16 y 17: preguntas elaboradas por el grupo investigador y que evaluaban sexo, edad, estado civil, grado de instrucción, ocupación, domicilio fijo, uso de drogas, orientación sexual, efectos adversos y motivos de cumplimentación incorrecta.

- Preguntas 10 al 15: correspondieron al cuestionario de adherencia SMAQ.

- Pregunta 18: correspondieron al Cuestionario de salud general de Goldberg, que evaluaba morbilidad psíquica.

- Pregunta 19: correspondió al cuestionario de Apoyo social funcional de Duke, que evaluaba apoyo social.

- Pregunta 20: correspondió al índice de calidad de vida, que evaluaba calidad de vida relacionada a la salud.

Durante los meses de julio a diciembre de 2007, se invitó a participar a todos los pacientes infectados con el VIH en Targa que asistieron al servicio de Infectología del HNAL; previo consentimiento informado procedieron al autollenado del cuestionario estructurado. Para conocer el estadío de la enfermedad, el recuento de células CD4 y el esquema de tratamiento (número total de pastillas al día, frecuencia de dosis, tiempo en tratamiento), se revisó los registros del servicio de Infectología del HNAL y se vertió los datos en una ficha de recolección confeccionada para el estudio.

Se elaboró una base de datos con el programa SPSS versión 15,0, para el análisis de los datos. Se determinó la frecuencia de todas las variables estudiadas. Posteriormente, la relación entre las variables independientes y la no adherencia fue contrastada mediante la prueba chi cuadrado para las variables cualitativas y con la prueba $T$ para las cuantitativas. Para el análisis multivariado, se realizó 
un análisis de regresión logística. Como medida de asociación, se utilizó el OR con IC 95\%; se estableció un nivel de significación estadística $p<0,05$.

\section{RESULTADOS}

Se encuestó 472 personas; se excluyó 7 encuestas, debido al llenado inadecuado de la misma, obteniendo un muestra final de 465. La edad de los pacientes varió entre 19 y 82 años, con una edad promedio de 36,8 (DE \pm 9,1); 64,1\% (298) era varón y 35,9\% (167) mujer; 28\% de los encuestados carecía de domicilio fijo. Al evaluar calidad de vida relacionada a la salud con el Índice de calidad de vida, 20,6\% de los encuestados tenía un nivel bajo de calidad de vida. Según el cuestionario de Apoyo social funcional de Duke, 28\% presentó apoyo social bajo. Al evaluar la morbilidad psíquica con el Cuestionario de salud general de Goldberg, se encontró que estaba presente en $36,1 \%$ de los encuestados. Solo $54(11,6 \%)$ encuestados refirieron haber consumido algún tipo de droga en los últimos seis meses, siendo la droga más frecuentemente consumida el alcohol, en 35 encuestados $(64,8 \%)$, el tabaco en seis $(11,1 \%)$, la cocaína y marihuana en cinco $(9,3 \%)$, respectivamente, y cocaína en tres $(5,5 \%)$. Respecto a la presencia de síntomas o molestias en el último mes, encontramos que 85,6\% mencionó haberlos tenido. Las demás características son mostradas en la tabla 1 .

Al evaluar la adherencia, 35,9\% de los encuestados resultó ser no adherente a su tratamiento antirretroviral (IC95\% 31,6 a 40,5), distribuyéndose en 59 mujeres $(35,3 \%)$ y 108 varones $(64,7 \%)$. El principal motivo de no adherencia referido por los pacientes fue el olvido de la toma del medicamento $(64,1 \%)$, seguido de dificultad horaria $(11,4 \%)$ (tabla 2 ).

Respecto al tratamiento antirretroviral, el número de pastillas que deben tomar diariamente varió entre 2 y 10 (promedio 2,9 $\pm 1,9$ ), siendo la frecuencia de toma dos veces al día en todos los encuestados. El tiempo que recibían Targa varió entre 1,1 y 46,3 meses (promedio $15,6 \pm 10,9)$. Todos los pacientes estuvieron en estadio sida. El recuento de CD4

Tabla1. Características de los adultos infectados con el VIH-sida.

\begin{tabular}{|c|c|c|c|}
\hline Características & Frecuencia & Porcentaje & $\mathrm{IC} 95 \%$ \\
\hline \multicolumn{4}{|l|}{ Sexo } \\
\hline Femenino & 167 & 35,9 & $31,6-40,5$ \\
\hline Masculino & 298 & 64,1 & $59,5-68,4$ \\
\hline \multicolumn{4}{|l|}{ Estado civil } \\
\hline Unidos (casado, conviviente) & 180 & 38,7 & $34,3-43,3$ \\
\hline No unidos (divorciado/separado, soltero, viudo) & 285 & 61,3 & $56,7-65,7$ \\
\hline \multicolumn{4}{|l|}{ Grado de instrucción } \\
\hline Ninguno/primaria & 110 & 23,6 & $19,9-27,8$ \\
\hline Secundaria & 224 & 48,2 & $43,6-52,8$ \\
\hline Superior & 131 & 28,2 & $24,2-32,5$ \\
\hline \multicolumn{4}{|l|}{ Ocupación } \\
\hline Trabajador independiente & 210 & 45,2 & $40,6-49,8$ \\
\hline Su casa & 97 & 20,9 & $17,3-24,9$ \\
\hline Empleado & 52 & 11,2 & $8,5-14,5$ \\
\hline Obrero & 50 & 10,7 & $8,2-14,0$ \\
\hline Trabajadora del hogar & 22 & 4,7 & $3,1-7,2$ \\
\hline Estudiante & 21 & 4,5 & $2,9-6,9$ \\
\hline Otra ocupación & 13 & 2,8 & $1,6-4,9$ \\
\hline \multicolumn{4}{|l|}{ Orientación sexual } \\
\hline Homosexual/bisexual & 158 & 34,0 & $29,7-38,5$ \\
\hline Heterosexual & 307 & 66,0 & $61,5-70,3$ \\
\hline \multicolumn{4}{|l|}{ Domicilio fijo } \\
\hline Sí & 335 & 72,0 & $67,7-76,0$ \\
\hline No & 130 & 28,0 & $24,0-32,3$ \\
\hline \multicolumn{4}{|l|}{ Calidad de vida relacionada a la salud } \\
\hline Alta & 369 & 79,4 & $75,3-82,9$ \\
\hline Baja & 96 & 20,6 & $17,1-24,7$ \\
\hline \multicolumn{4}{|l|}{ Apoyo social } \\
\hline Alto & 335 & 72,0 & $67,7-76,0$ \\
\hline Bajo & 130 & 28,0 & $24,0-32,3$ \\
\hline \multicolumn{4}{|l|}{ Morbilidad psíquica } \\
\hline Sí & 168 & 36,1 & $31,8-40,7$ \\
\hline No & 297 & 63,9 & $59,3-68,2$ \\
\hline \multicolumn{4}{|l|}{ Consumo de drogas en los últimos 6 meses } \\
\hline Sí & 54 & 11,6 & $8,9-15,0$ \\
\hline No & 411 & 88,4 & $85,0-91,1$ \\
\hline \multicolumn{4}{|l|}{ Síntomas/molestias en el último mes } \\
\hline Sí & 398 & 85,6 & $82,0-88,6$ \\
\hline No & 67 & 14,4 & $11,4-18,0$ \\
\hline
\end{tabular}

varió entre 11 y 713 células (promedio de $262,7 \pm 142,1)$.

En el análisis bivariado, se observó que los factores asociados a la no adherencia fueron: ser homosexual/bisexual (OR 1,95, IC95\% 1,31 a 2,89, $p=0,001)$, tener una baja CVRS (OR 5,84, IC95\% 3,58 a $9,51, p<0,001$ ), apoyo social bajo (OR $4,15$, IC95\% 2,71 a 6,35, $p<0,001)$, no tener domicilio fijo (OR 1,99, IC95\% $1,32$ a 3,02, $p=0,001)$ y presentar morbilidad psíquica (OR 2,69, IC95\% 1,81 a $3,99, p<0,001$ ) (tabla 3). Los pacientes no adherentes al Targa presentaron una edad promedio menor que los pacientes encontrados adherentes $(36,0 \pm 9,6$ vs. $37,2 \pm 8,9)$ y tomaban un mayor número de pastillas al día que los adherentes $(3,1$ $\pm 2,2$ vs. $2,7 \pm 1,8)$. Sin embargo, estas diferencias no fueron estadísticamente significativas ( $p=0,16$ y 0,07$)$. Los pacientes no adherentes a su tratamiento tenían un mayor tiempo recibiendo el tratamiento que los adherentes $(17,9$ $\pm 9,8$ vs. $14,2 \pm 10,6)$, siendo esta diferencia estadísticamente significativa $(p=0,0003)$.

Tras el análisis multivariado, las variables que resultaron independientemente asociadas a la no adherencia fueron: ser homosexual/bisexual (OR 3,85, IC95\% $1,98$ a $7,51, p<0,001)$, tener una baja calidad de vida relacionada a la salud (OR 6,22, IC95\% 3,47 a 11,13, p<0,001), 
Tabla 2. Motivo de no adherencia de los adultos infectados con el VIH-sida.

\begin{tabular}{lccc}
\hline \multicolumn{1}{c}{ Motivo de no adherencia } & Frecuencia & Porcentaje & IC95\% \\
\hline Olvido & 107 & 64,1 & $56,3-71,3$ \\
Dificultad horaria & 19 & 11,3 & $7,0-17,2$ \\
Creo que los medicamentos tienen demasiados & & & \\
efectos negativos & 8 & 4,8 & $2,1-9,2$ \\
No tengo información suficiente sobre ellos & 8 & 4,8 & $2,1-9,2$ \\
Conozco a gente que no le ha ido bien & 5 & 3,0 & $1,0-6,8$ \\
No me gusta tomar medicinas & 5 & 3,0 & $1,0-6,8$ \\
No he soportado los efectos secundarios & & & \\
del medicamento & 2 & 1,2 & $0,1-4,3$ \\
Voluntad propia & 2 & 1,2 & $0,1-4,3$ \\
Otro motivo & 11 & 6,6 & $3,3-11,5$ \\
Total & 167 & 100,0 & \\
\hline
\end{tabular}

apoyo social bajo (OR 5,41, IC95\% 3,17 a $9,22, p<0,001)$, no tener domicilio fijo (OR 3,34, IC95\% 1,93 a 5,79, p<0,001), tener morbilidad psíquica (OR 2,93, IC95\% 1,78 a $4,82, p<0,001)$, y tener mayor tiempo en tratamiento (OR 1,04, IC95\% 1,02 a 1,07, p=0,002) (tabla 4).

Tabla 3. Factores asociados a la falta de adherencia al Targa.

\begin{tabular}{|c|c|c|c|c|c|}
\hline \multirow[t]{2}{*}{ Variable } & \multicolumn{2}{|c|}{ Adherencia } & \multirow[t]{2}{*}{ OR } & \multirow[t]{2}{*}{ IC 95\% } & \multirow[t]{2}{*}{$p$} \\
\hline & No & Sí & & & \\
\hline \multicolumn{6}{|l|}{ Sexo } \\
\hline Femenino & 59 & 108 & 096 & $0,65-1,43$ & 0,924 \\
\hline Masculino & 108 & 190 & - & - & \\
\hline \multicolumn{6}{|l|}{ Estado civil } \\
\hline No unidos & 110 & 175 & 1,36 & $0,91-2,01$ & 0,129 \\
\hline Unidos & 57 & 123 & - & - & \\
\hline \multicolumn{6}{|l|}{ Grado de instrucción } \\
\hline Ninguno/primaria & 44 & 66 & 1,09 & $0,79-1,50$ & 0,496 \\
\hline Secundaria & 75 & 149 & 0,91 & $0,68-1,22$ & \\
\hline Superior & 48 & 83 & - & - & \\
\hline \multicolumn{6}{|l|}{ Orientación sexual } \\
\hline Bisexual/homosexual & 73 & 85 & 1,95 & $1,31-2,89$ & 0,001 \\
\hline Heterosexual & 94 & 213 & - & - & \\
\hline \multicolumn{6}{|c|}{ Calidad de vida relacionada a la salud } \\
\hline Baja & 66 & 30 & 5,84 & $3,58-9,51$ & $<0,001$ \\
\hline Alta & 101 & 268 & - & - & \\
\hline \multicolumn{6}{|l|}{ Apoyo social } \\
\hline Bajo & 78 & 52 & 4,15 & $2,71-6,35$ & $<0,001$ \\
\hline Alto & 89 & 246 & - & - & \\
\hline \multicolumn{6}{|c|}{ Consumo de droga en los últimos 6 meses } \\
\hline Sí & 24 & 30 & 1,50 & $0,84-2,66$ & 0,215 \\
\hline No & 143 & 268 & - & - & \\
\hline \multicolumn{6}{|l|}{ Domicilio fijo } \\
\hline No & 62 & 68 & 1,99 & $1,32-3,02$ & 0,001 \\
\hline Sí & 105 & 230 & - & - & \\
\hline \multicolumn{6}{|l|}{ Morbilidad psíquica } \\
\hline Sí & 85 & 83 & 2,69 & $1,81-3,99$ & $<0,001$ \\
\hline No & 82 & 215 & - & - & \\
\hline \multicolumn{6}{|c|}{ Síntomas/molestias en el último mes } \\
\hline Sí & 144 & 254 & 1,08 & $0,63-1,87$ & 0,877 \\
\hline No & 23 & 44 & - & - & \\
\hline
\end{tabular}

OR: odds ratio; IC 95\%: intervalo de confianza del $95 \%$. les, aunque cabe mencionar que han sido realizados en poblaciones con características diversas. Se ha empleado diferentes diseños de estudio y se ha cuantificado la adherencia en forma variada, por lo que debemos tener en cuenta estas diferencias al analizarlos. Los estudios longitudinales realizados permiten relacionar la adherencia con la evolución clínica y emplean métodos más objetivos para medirla. Sin embargo, las muestras son más pequeñas y el tiempo de seguimiento es variado, lo que dificulta aún más su comparación. En los estudios transversales, encontramos muestras más numerosas y generalmente se valora la adherencia mediante autocuestionarios estandarizados; además, los resultados de prevalencia de no adherencia son más homogéneos.

A pesar de los estudios realizados, no se ha establecido cuál es el método idóneo para valorar la adherencia a los antirretrovirales ${ }^{(12,19,21)}$. En el presente estudio se empleó la autodeclaración del paciente mediante cuestionario (cuestionario SMAQ), tanto por su simplicidad, costo bajo y porque es el método que ha presentado menos variación en sus resultados ${ }^{(19)}$. Además, teniendo en consideración la sensibilidad baja de los cuestionarios y la recomendación de usar métodos asociados ${ }^{(12,15)}$, se consideró la evaluación del Registro de dispensación del servicio de Infectología del HNAL. Con estos métodos, se encontró una prevalencia de no adherencia de $35,9 \%$, la cual es similar a la mayoría de estudios transversales que emplean la autodeclaración del paciente asociado con el registro de dispensación o el recuento de comprimidos, para la valoración de la adherencia, cuyos resultados varían entre 29 y $45 \%$ de prevalencia de no adherencia ${ }^{(7,9,13,15,24)}$. Existen estudios donde la prevalencia encontrada es menor ( 9 a $19 \%)$, probablemente debido a que son estudios longitudinales y utilizan los sistemas MEMS para valorar la adherencia ${ }^{(8,25,26)}$. Un estudio realizado anteriormente en esta misma población mostró una prevalencia de no adherencia de 19,8\%, la cual es menor a la encontrada en este estudio. Esto puede deberse a que en ese estudio se evaluó la adherencia a los 6 meses de iniciado el tratamiento, por lo que al ser pacientes recién ingresados al 
Tabla 4. Factores asociados a la no adherencia al Targa. Análisis de regresión logística.

\begin{tabular}{|c|c|c|c|}
\hline Variable & OR & IC 95\% & $p$ \\
\hline \multicolumn{4}{|l|}{ Sexo } \\
\hline Femenino & 1,19 & $0,66-2,16$ & 0,568 \\
\hline Masculino & - & - & \\
\hline \multicolumn{4}{|l|}{ Estado civil } \\
\hline No unidos & 1,37 & $0,80-2,37$ & 0,258 \\
\hline Unidos & - & - & \\
\hline \multicolumn{4}{|l|}{ Grado de instrucción } \\
\hline Ninguno/primaria & 0,51 & $0,22-1,22$ & 0,130 \\
\hline Secundaria & 0,62 & $0,35-1,10$ & 0,102 \\
\hline Superior & & - & - \\
\hline \multicolumn{4}{|l|}{ Orientación sexual } \\
\hline Bisexual/homosexual & 3,85 & $1,98-7,51$ & $<0,001$ \\
\hline Heterosexual & - & - & \\
\hline \multicolumn{4}{|c|}{ Calidad de vida relacionada a la salud } \\
\hline Baja & 6,22 & $3,47-11,13$ & $<0,001$ \\
\hline Alta & - & - & \\
\hline \multicolumn{4}{|l|}{ Apoyo social } \\
\hline Bajo & 5,41 & $3,17-9,22$ & $<0,001$ \\
\hline Alto & - & - & \\
\hline \multicolumn{4}{|c|}{ Consumo de droga en los últimos 6 meses } \\
\hline Sí & 2,37 & $0,87-6,47$ & 0,091 \\
\hline No & - & - & \\
\hline \multicolumn{4}{|l|}{ Domicilio fijo } \\
\hline No & 3,34 & $1,93-5,79$ & $<0,001$ \\
\hline Sí & - & - & \\
\hline \multicolumn{4}{|l|}{ Morbilidad psíquica } \\
\hline Sí & 2,93 & $1,78-4,82$ & $<0,001$ \\
\hline No & - & - & \\
\hline \multicolumn{4}{|c|}{ Síntomas/molestias en el último mes } \\
\hline Sí & 1,05 & $0,51-2,19$ & 0,895 \\
\hline No & - & - & \\
\hline Edad & 0,99 & $0,97-1,02$ & 0,795 \\
\hline Tiempo en Targa & 1,04 & $1,02-1,07$ & 0,002 \\
\hline Número de pastillas al día & 1,09 & $0,96-1,23$ & 0,190 \\
\hline
\end{tabular}

OR: odds ratio; IC 95\%: intervalo de confianza del 95\%.

programa tuvieron un mayor control de su terapéutica ${ }^{(23)}$.

Respecto a las variables estudiadas, no se encontró que la falta de adherencia esté relacionada con la edad o el sexo del paciente. Sin embargo, existen estudios que establecen que a mayor edad hay más probabilidad de ser adherente ${ }^{(8,9,14)}$, definiéndose el ser joven como un factor de riesgo para ser no adherente ${ }^{(27)}$. Se ha descrito también que las mujeres tienden a ser menos adherentes que los hombres (25). En forma general, se ha establecido que las variables demográficas no tienen un rol determinante en la adherencia al tratamiento antirretroviral ${ }^{(12)}$. Por otro lado, se encontró que las personas que son homosexuales o bisexuales tienen más riesgo de ser no adherentes que los heterosexuales. Al revisar la literatura, vemos que existen resultados contradic- estudios manifiestan que es un factor claramente relacionado con la adherencia ${ }^{(7,12,25)}$. En este estudio, $85,6 \%$ de los encuestados manifestó haber presentado durante el último mes alguna sintomatología relacionada con el consumo de los fármacos, pero no se encontró que esta variable tuviera una asociación significativa con la no adherencia. Del mismo modo, el consumo activo de drogas no resultó estar asociado significativamente, lo cual difiere con la literatura encontrada $(9,18,25,28,31,32)$.

Se ha observado que algunas patologías de carácter psicológico tienen un papel importante en el cumplimiento de la adherencia; se ha visto que la ansiedad, la depresión y el estrés están asociados al incumplimiento en el tratamiento. Una forma de denominar en forma general estas patologías es bajo el término de morbilidad psíquica. $\mathrm{Al}$ igual que en nuestro estudio, existe evidencia que respalda que presentar morbilidad psíquica es un factor de riesgo para ser no adherente $(9,14,27,28)$. De igual forma, el presentar una CVRS baja ${ }^{(12,14,33)}$ y tener apoyo social bajo ${ }^{(9,27,29,34)}$ estuvieron relacionados con la falta de adherencia a la terapia antirretroviral.

En general, todo paciente en Targa registrado en una institución de salud, sea local, nacional o internacional, provee una dirección para efectos de control y monitoreo del cumplimiento de la terapia. Sin embargo, 28\% de los encuestados refirió no tener un domicilio fijo, coincidiendo con la literatura, al encontrar que no tener domicilio fijo es un factor de riesgo para ser no adherente a la terapia antirretroviral ${ }^{(25,27,34)}$.

Podemos concluir que en nuestra población la prevalencia encontrada fue similar a la descrita en la mayoría de estudios, a pesar de la heterogeneidad ya mencionada, teniendo en cuenta que resultó mayor a la encontrada previamente es este mismo hospital. Además, los factores independientemente asociados a la falta de adherencia fueron: ser homosexual/ bisexual, tener CVRS baja, apoyo social bajo, no tener domicilio fijo, presentar morbilidad psíquica, tener mayor tiempo recibiendo el tratamiento y tomar mayor número de pastillas al día; observando 
que los factores de carácter psicosocial son los que influyeron de manera más importante en la adherencia, seguidos de los factores relacionados al tratamiento antirretroviral; los factores demográficos son los que mostraron no tener influencia alguna en la adherencia al tratamiento.

\section{AGRADECIMIENTOS}

Al personal del servicio de Infectología del Hospital Nacional Arzobispo Loayza, por el apoyo y facilidades brindadas para el desarrollo del presente estudio; especialmente, al Dr. Carlos Benites Villafane, médico asistente del servicio, por las sugerencias y recomendaciones dadas. Asimismo, agradecemos a nuestros compañeros Marina Egoávil Guerra, Joseph Castillo Cuenca y Jack Pinto Venero, por su valiosa colaboración en el desarrollo de esta investigación.

\section{REFERENCIAS BIBLIOGRÁFICAS}

1. MINSA-DGE. Análisis de la situación epidemiológica del VIH/SIDA en el Perú: bases epidemiológicas para la prevención y control. Lima: Minsa; 2006.

2. ONUSIDA/OMS. Situación de la epidemia de SIDA. Ginebra: Onusida; 2007.

3. Ministerio de Salud, Oficina General de Epidemiología. Situación del VIH/SIDA en el Perú. Casos de SIDA según año de diagnóstico. Perú, 1983 - 2007. Boletín Epidemiológico [Internet]. Diciembre 2007. Disponible en: http://www.dge. gob.pe/vigilancia/vih/Boletin_2008/diciembre. pdf

4. Cabrera D, Sánchez S, Jave 0, Carrión M, Jamanca R. Infección VIH/Sida en la Jurisdicción de la Dirección de Salud Lima Ciudad, 1984 - 2004. Rev Peru Med Exp Salud Pública. 2005;22(4):274-80.

5. Norma Técnica para la adherencia al tratamiento antirretroviral de gran actividad (TARGA) en adultos infectados por el virus de la inmunodeficiencia humana. Lima: Minsa; 2004.

6. Guerra L, Parras F. La adherencia a los antirretrovirales: una tarea de todos. Enf Emerg. 2000;2(1):5-9.

7. Pinheiro CA, de-Carvalho-Leite JC, Drachler ML, Silveira VL. Factors associated with adherence to antiretroviral therapy in HIV/AIDS patients: a cross-sectional study in Southern Brazil. J Med Biol Res. 2002;35(10):1173-81.

8. Hinkin C, Hardy D, Mason K, Castellón S, Durvasula R, Lam M, et al. Medication adherence in HIV-infected adults: effect of patient age, cognitive status, and substance abuse. AIDS. 2004;18(1):19-25.

9. Gordillo V, Del Amo J, Soriano V, González-Lahoz J. Sociodemographic and psychological variables influencing adherence to antiretroviral therapy. AIDS. 1999;13:1763-9.

10. Günthard HF, Wong Jk, Ingnacio CC, Guatelli JC, Riggs NL, Havlir DV, Richman DD. Human immunodeficiency virus replication and genotypic resistance blood and lymph nodes after a year of potent antiretroviral therapy. J Virol. 1998;72:2422-8.

11. Kaufman D, Pantaleo G, Sudre P. CD4-cell count in HIV infected individuals remaining viraemic with highly active antiretroviral therapy (HAART). Lancet. 1998;351:723-4.

12. Knobel H, Escobar I, Polo R, Ortega L, MartínConde T, Casado JL, et al. Recomendaciones GESIDA/SEFH/PNS para mejorar la adherencia al tratamiento antirretroviral en el año 2004. Enferm Infecc Microbiol Clin. 2005;23(4):221-31.

13. Ladero L, Orejudo S, Carrobles JS. Variables psicosociales en la adherencia al tratamiento antirretroviral en pacientes adscritos a un programa de mantenimiento con metadona. Psicothema. 2005;17(4):575-81.

14. Ruiz-Pérez I, Labry-Lima A0, Prada-Pardal JL, Rodríguez-Baño J, Causse-Prados M, López-Ruz MA, et al. Impacto de los factores demográficos y psicosociales en la no adherencia a los fármacos antirretrovirales. Enferm Infecc Microbiol Clin. 2006;24(6):373-8.

15. Puigventós F, Riera M, Delibes C, Peñaranda M, De la Fuente L, Boronat A. Estudios de adherencia a los fármacos antiretrovirales. Una revisión sistemática. Med Clin (Barc). 2002;119(4):130-7.

16. Reyero GC. Sida: factores psico-sociales en la adherencia al tratamiento. Actualizaciones en SIDA. 2005;13(48):43-55. Disponible en: http:// www.huesped.org.ar/site/secciones/publicaciones/ actualizaciones/pdfs/48/Reyero.pdf

17. Knobel H, Alonso J, Casado JL, Collazos J, Gonzalez J, Ruiz I, Kindelan JM,Carmona A, Juega J, Ocampo A. Validation of a simplified medication adherence questionnaire in a large cohort of HIV-infected patients: the GEEMA Study. AIDS. 2002;16(4):60513.

18. Wendel CS, Mohler MJ, Kroesen K, Ampel NM, Gifford AL, Coons SJ. Barriers to use of electronic adherence monitoring in an HIV clinic. Ann Pharmacother. 2001;35(9):1010-5.

19. Liu H, Golin CE, Miller LG, Hays RD, Beck CK, Sananndaji S, et al. A comparison study of multiple measures of adherence to HIV protease inhibitors. An Intern Med. 2001;134:968-77.

20. Piña López JA, Corrales Rascón AE, Mungaray Padilla K, Valencia Vidrio MA. Instrumento para medir variables psicológicas y comportamientos de adhesión al tratamiento en personas seropositivas frente al VIH (VPAD-24). Rev Panam Salud Publica. 2006;19(4):217-28.

21. Codina C, Martínez M, Tuset M, Del Cacho E, Martín MT, Miró JM, et al. Comparación de tres métodos de cálculo de adherencia en pacientes con tratamiento antirretroviral. Enferm Infecc Microbiol Clin. 2002;20(10):484-90.

22. Informe de la ESN PCITS/VIH/SIDA. Lima: Minsa; 2005.

23. Benites C, Cabrian K, Pinedo Y, Matos E, Angles E, Zapana E, et al. Eficacia, seguridad y adherencia a la terapia antirretroviral de gran actividad (TARGA) en el Hospital Nacional Arzobispo Loayza. Rev Peruana Enf Infec Trop. 2005;4(5):24-6.

24. Mills EJ, Nachega JB, Buchan I, Orbinski J, Attaran A, Singh $S$, et al. Adherence to antiretroviral therapy in sub-Saharan Africa and North America: a metaanalysis. JAMA. 2006;296:679-90.

25. Berg KM, Demas PA, Howard AA, Schoenbaum EE, Gourevitch MN, Arnsten JH. Gender differences in factors associated with adherence to antiretroviral therapy. J Gen Intern Med. 2004;19(11):1111-7.

26. Laniece I, Ciss M, Desclaux A, Diop K, Mbodj $F$, et al. Adherence to HAART and its principal determinants in a cohort of Senegalese adults. AIDS. 2003;17(3):103-8.

27. Mehta S, Moore RD, Graham NMH. Potential factors affecting adherence with HIV therapy. AIDS. 1997;11(14):1665-70.

28. Gebo KA, Keruly J, Moore RD. Association of social stress, illicit drug use, and health beliefs with nonadherence to antiretroviral therapy. J Gen Intern Med. 2003;18:104-11.

29. Ammassari A, Trotta M, Murri R, Castelli F, Narciso $P$, Noto $P$, et al. Correlates and predictors of adherence to highly active antiretroviral therapy: overview of published literature. I Acquir Immune Defic Syndr. 2002;31(3):123-7.

30. Kleeberger CA, Phair JP, Strathdee SA, Detels $R$, Kingsley L, Jacobson LP. Determinants of heterogeneous adherence to HIV-antiretroviral therapies in the Multicenter AIDS Cohort Study. J Acquir Immune Defic Syndr. 2001;26(1):82-92.

31. Castro A. Adherence to antiretroviral therapy: Merging the clinical and social course of AIDS. PLoS Med. 2005;2(12):1217-21.

32. Martín-Sánchez V, Ortega-Valín L, Pérez-Simón MR, Mostaza-Fernández JL, Ortiz de UrbinaGonzález JJ, Rodríguez Marín M, et al. Factores predictores de no adherencia al tratamiento antirretroviral de gran actividad. Enferm Infecc Microbiol Clin. 2002;20(10):491-7.

33. Holzemer WL, Corless IB, Nokes KM, Turner JG, Brown MA, Powell-Cope GM, et al. Predictors of selfreported adherence in persons living with HIV disease. AIDS Patient Care STD. 1999;13:18597.

34.Duran S, Spire B, Raffi F, Walter V, Bouhour D, Journot $V$, et al. Self-reported symptoms after initiation of a protease inhibitor in HIV-infected patients and their impact on adherence to HAART. HIV Clin Trials. 2001;2:38-45.

Manuscrito recibido el 27 de agosto de 2009 y aceptado para publicación el 5 de noviembre de 2009.

Correspondencia:

Oscar Roberto Alvis Huamán

Av. Brasil 2760

Lima 21, Perú

Correo-e: oscaralvis4@hotmail.com 